\title{
QoS Support for Cross-Layer Scheduling Algorithm in Wireless Networks
}

\author{
K. Raghavendra Rao ${ }^{1}$, Vadipina Amarnadh ${ }^{2}$ \\ ${ }^{1,2}$ Assistant Professor, Department of CSE, Anurag group of Institutions, Hyderabad, Telangana, India
}

\begin{abstract}
Scheduling plays an crucial role in providing quality of service (QoS) support to multimedia communications in different kinds of wireless networks, including wireless sensor networks, cellular networks and mobile ad hoc networks. The authors proposed a scheduling algorithm at the medium access control (MAC) layer for multiple connections with diverse QoS requirements, where every connection employs adaptive modulation and coding (AMC) scheme at the physical (PHY) layer over wireless fading channels. Each connection is assigned with a priority, which is updated dynamically based upon its channel and service status; the connection with highest priority is scheduled every time. The authors scheduler provides diverse QoS guarantees, uses wireless bandwidth efficiently, and enjoys scalability, flexibility and low implementation complexity. Its performance cab be evaluated via simulations.
\end{abstract}

Keywords: Cross-layer design, IEEE 802.16, quality of service (QoS), scheduling algorithm, Adaptive modulation and coding (AMC), wireless networks

\section{Introduction}

The Rapid growth in demand for high-quality and high-speed multimedia communications is creating challenges and opportunities for next-generation wired and wireless network designs. Multimedia communications involves diverse quality of service (QoS) requirements for different number of applications including data, voice and real time, or streaming video/audio.For future wireless networks providing QoS guaranteed services is necessary, including cellular networks, mobile ad hoc networks, and some wireless sensor networks like IEEE 802.11, IEEE 802.16 and IEEE 802.15 standard wireless networks. Such networks are visualized to support multimedia services with different QoS requirements. However, the preceding standards defines only signaling and QoS architecture, but it do not specify the scheduling algorithm that will ultimately provide QoS support. Scheduling plays a crucial role in QoS provision. There are many traffic scheduling algorithms that are available for wireline networks [25], that cannot be directly applied to wireless networks because of fundamental differences between them. For example, traditional schedulers for wireline networks only consider traffic and queuing status; whereas, channel capacity in wireless networks is time changing due to Doppler effects and multipath fading. To schedule wireless resources like bandwidth and power efficiently for diverse QoS guarantees, the associated queuing behavior induced by heterogenous traffic as well as dynamic variation of wireless channel should be considered on scheduler design.

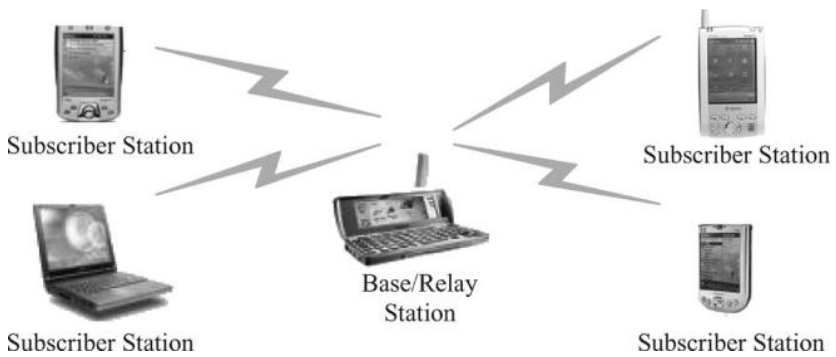

Figure 1: Network topology.
Here, we introduced a priority-based scheduler at the medium access control (MAC) layer for multiple connections with diverse QoS requirements, where each connection can employs adaptive modulation and coding (AMC) scheme at the physical layer. We propose a priority function (PRF) for each connection admitted in the system and update it dynamically depending on the QoS satisfaction, wireless channel quality and service priority across the layers. Thus, the connection with the highest priority is scheduled every time. Our scheduler provides authorized QoS guarantees and utilizes the wireless bandwidth efficiently while enjoying with low implementation flexibility, complexity and scalability.

\section{System Architecture}

\subsection{Network Configuration}

The wireless network topology under consideration is defined above. Multiple subscriber stations (SS) are connected to the relay station or base station (BS) over wireless channels, where multiple connections sessions, flows can be supported by each subscriber stations. This kind of star topology is not only applicable for cellular networks but also used to show the connections between each relay station and multiple subscriber stations in mobile adhoc networks and wireless sensor networks.

All connections can communicate with base station using time division multiplexing/time division multiple access (TDM/TDMA). We can focus on the downlink although our results can be extended to the uplink as well. The wireless link of each connection from the base station to each subscriber station is shown below fig 2. A buffer is implemented at the base station for each connection and operates in a first input first output (FIFO) mode. The AMC controller follows the buffer at the base station (transmitter), and the AMC selector is implemented at the subscriber station (receiver). 


\section{International Journal of Science and Research (IJSR) ISSN (Online): 2319-7064}

Index Copernicus Value (2013): 6.14 | Impact Factor (2015): 6.391

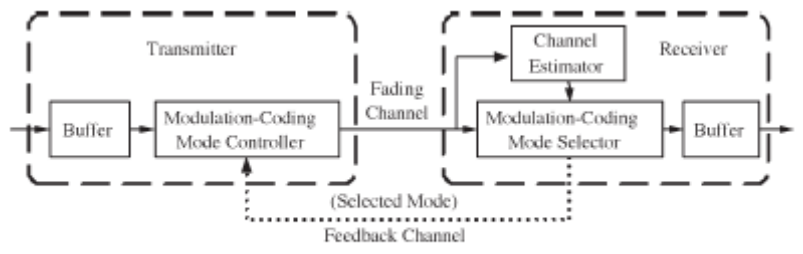

Fig. 2. Wireless link from BS to SS.

At the physical layer, multiple transmission modes are available for each user, with each mode representing pair of a specific modulation format and forward error control (FEC) code, as in IEEE 802.11/15/16, 3GPP, and 3GPP2 standards. Based on those channel estimates obtained at receiver, the AMC selector determines the modulationcoding pair (mode or burst profile), whose index is sent back to transmitter through a feedback channel, for AMC controller to update transmission mode. Soft decision and Coherent demodulation Viterbi decoding are employed at the receiver side. The decoded bit streams are mapped to packets, which can pushed upward to MAC.

We consider the following group of transmission modes as in IEEE 802.16 standard [3].

Transmission modes (TM): The modulations are Mn-ary rectangular/square quadrature amplitude modulators (QAMs), and FEC codes are Reed-Solomon (RS) concatenated with convolutional codes (CC) (see Table I). Although, we have focus on this TM, other transmission modes can be similarly to be constructed [1]-[3], [13].

At the physical layer, the processing unit is a frame containing of multiple transmitted symbols. At the MAC layer, the processing unit is an packet comprising multiple information bits. Fig. 3 details about the packet and frame structures.

1)At the MAC layer, each packet consists a fixed number of bits $\mathrm{Nb}$, which can include payload, cyclic redundancy check (CRC) bits and packet header. After modulating and coding with mode $\mathrm{n}$ of rate $\mathrm{Rn}$ as in Table I, each packet is mapped with a symbol block containing $\mathrm{Nb} / \mathrm{Rn}$ symbols.

2) At the Physical layer, the data are transmitted in frame by frame through wireless channel, with each frame containing the fixed number of symbols Ns. Given a fixed symbol rate, the frame duration Tf (in seconds) is said to be constant and represents the time unit. With TDM, each frame is to be divided into $\mathrm{Nd}+\mathrm{Nc}$ time slots, where for convenience we kept each time slot contain a fixed number of $2 \mathrm{Nb} / \mathrm{R} 1$ symbols. As a result of this, each time slot can transmit exactly 2Rn/R1 packets with transmission mode n. For TM in particular, one time slot can be accommodate $2 \mathrm{R} 1 / \mathrm{R} 1=2$ packets with mode $\mathrm{n}=1,2 \mathrm{R} 2 / \mathrm{R} 1=3$ packets with mode $n=2$, and so on. The Nc time slots contains control information and pilots. The Nd time slots convey data, which are scheduled dynamically with different connections. Each connection is allocated with certain number of time slots during each frame.

\subsection{QoS Architecture at the MAC}

At the MAC, each connection belongs to single service class and it is associated with a set of QoS parameters that measures its characteristics. Four QoS classes are provided by MAC in the IEEE 802.16 standard.

1) At first, Unsolicited grant service supports constant bit rate (or) fixed throughput connections such as E1/T1 lines and voice over IP (VoIP). This services provides guarantees on latency, throughput, and jitter to the necessary levels as TDM services. The QoS metrics here are the service rate and packet error rate (PER).

2) 2)Secondly, Real-time polling service (RTPS) provides guarantees on latency and throughput, but with greater forbearance on latency relative to UGS, e.g., video streaming and MPEG video conferencing. The delayed packets are useless and it will be dropped. The QoS metrics are PER and the maximum delay.

3) Non-real-time polling service (nrtPS) provides guarantees in terms of throughput only and it is suitable for mission critical data applications, such as FTP. These applications are to be time insensitive and requires minimum throughput. For example, an FTP file can be downloaded with a bounded waiting time if the minimum reserved rate is to be guaranteed. The quality of sercive metrics are the PER and minimum reserved rate.

4) Best effort service provides no guarantees for delay or throughput and is used for Hypertext Transfer Protocol and electronic mail (e-mail), for example. BE applications will receives the residual bandwidth, after the bandwidth is allocated to the connections of the previous three service classes. Although, there is no delay and rate is specified for BE connections, a prescribed PER should be maintained over wireless communication channels.

The signaling and procedure for the service setup and maintenance of each connection are defined in the IEEE 802.16 standard. However, the IEEE standard does not defines the scheduling mechanism or traffic policing processes and admission control . The signaling overhead is not included in our analysis and design.

\subsection{AMC Design at the Physical Layer}

Efficient bandwidth utilization for a specific PER performance at the physical layer can be expert with AMC schemes, which match transmission parameters to timevarying wireless channel conditions flexible and have been adopted by many standard wireless networks, as IEEE 802.11/15/16 and 3GPP/3GPP2 [1]-[3], [7], [13].

Each connection with nrtPS, rtPS and BE services depends on AMC at the physical layer. The main objective of AMC is to maximize the data rate by adjusting transmission modes to channel variations which are maintaining a prescribed PER $\mathrm{P} 0$, and the design procedure is similar to that proposed in [5] and [16].

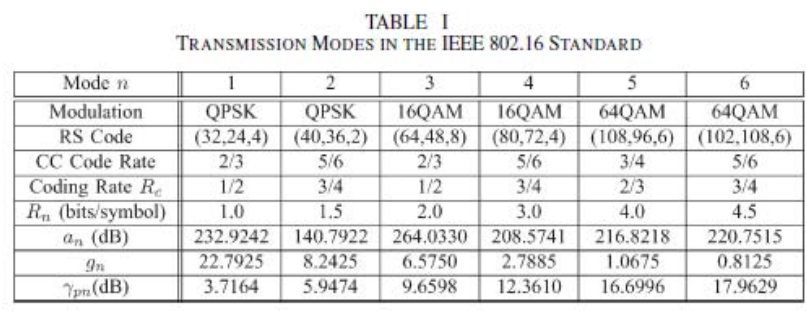


International Journal of Science and Research (IJSR)

ISSN (Online): 2319-7064

Index Copernicus Value (2013): 6.14 | Impact Factor (2015): 6.391

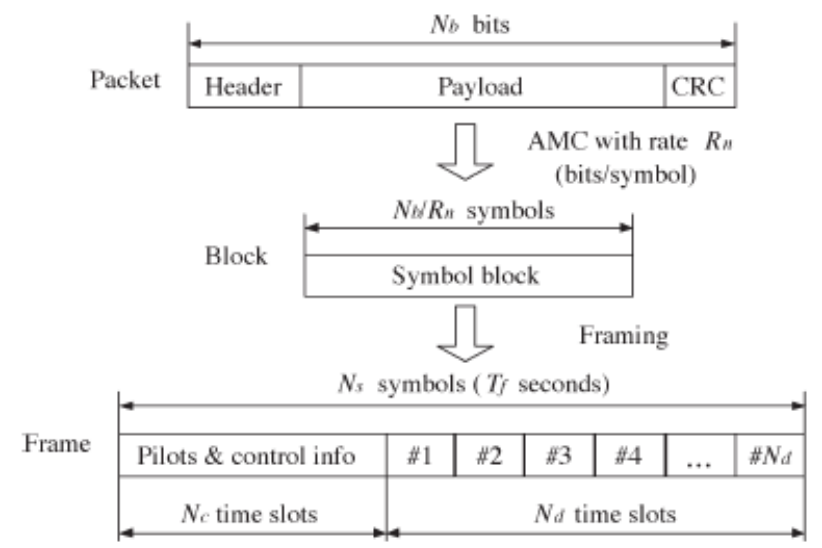

Fig. 3. Processing units at MAC and PHY.

Let $\mathrm{N}$, be denote the total number of transmission modes available ( $\mathrm{N}=6$ for $\mathrm{TM})$. As in [5], we can assume constant power transmission and partition the entire signal-to-noise ratio (SNR) range in $\mathrm{N}+1$ non-overlapping consecutive intervals, with boundary points can be denoted as $\{\gamma n\} \mathrm{N}+1$ $\mathrm{n}=0$. In this case, mode $n$ is chosen when $\gamma \in\left[\gamma_{n}, \gamma n+1\right)$,for $n=1, \ldots, N$. (1)

To avoid deep channel fades, no data can sent when $\gamma 0 \leq \gamma<$ $\gamma 1$, which corresponds to mode $\mathrm{n}=0$ with rate $\mathrm{R} 0=0$ bit/symbol. The main design objective of AMC is to decide the boundary points $\{\gamma \mathrm{n}\} \mathrm{N}+1 \mathrm{n}=0$.

To simplify the AMC design, we imprecise the PER expression in AWGN channels as

$$
\operatorname{PER}_{n}(\gamma) \approx \begin{cases}1, & \text { if } 0<\gamma<\gamma_{p n} \\ a_{n} \exp \left(-g_{n} \gamma\right), & \text { if } \gamma \geq \gamma_{p n}\end{cases}
$$

where $\mathrm{n}$ is mode index and $\gamma$ is received SNR. Parameters gn, an, and $\gamma \mathrm{pn}$ in (2) are mode-dependent and are obtained by fitting (2) to the exact PER via simulations presented in [4]. With packet length $\mathrm{Nb}=128$ bytes/packet, the fitting parameters for transmission modes in TM are available in Table I.

Using the approximate yet simple expression (2) easier the mode selection. This approach has also been adopted by Hole et al. [10], where bit error rate (BER) is used as figure of merit.

We set region boundary (switching threshold) $\gamma \mathrm{n}$ for the transmission mode $\mathrm{n}$ to be minimum SNR required to guarantee P0. Inverting the PER expression in (2), we obtain

$$
\begin{aligned}
\gamma_{0} & =0 \\
\gamma_{n} & =\frac{1}{g_{n}} \ln \left(\frac{a_{n}}{P_{0}}\right), \quad n=1,2, \ldots, N \\
\gamma_{N+1} & =+\infty .
\end{aligned}
$$

With the boundaries $\{\gamma n\} \mathrm{Nn}=0$ specified in (3), one can verify that the AMC in (1) can guarantees that PER is less than or equal to the P0. Maintaining the target PER performance, the proposed AMC transmissions with (1) and (3) are designed for maximize the spectral efficiency, with given finite transmission modes. In summary to that, our AMC design can guarantees that the PER is less than or equal to the P0 by determining $\{\gamma n\} \mathrm{Nn}=0$ as in (3) and updating the transmission mode as in (1).

\section{Scheduler Design}

In this section, we describe our scheduler for multiple connections with diverse QoS requirements.

\subsection{Scheduling UGS Connections}

In UGS services, the transmission mode at the physical layer is fixed to meet the specified average" PER requirement as in traditional TDMA cellular networks. For e.g., Global System for Mobile Communications (GSM). For example, the transmission mode would be selected in the initial service access phase via training to meet the average PER requirement. Therefore, the transmission mode is fixed during whole service time. The AMC design in above is not adopted for UGS connections because voice services may allow some instantaneous" packet loss and AMC feedback above should be reduced for low-rate voice traffic, e.g., 8kbps voice stream. For these specific reasons, the time slots allocated for UGS connections are fixed, based on constantrate requirements at the MAC.

We now denote the total time slots allocated to UGS connections as NUGS per frame. The residual time slots $\mathrm{Nr}$ $=\mathrm{Nd}-\mathrm{NUGS}$ are scheduled for the other three QoS classes, with UGS connections given higher priority than other three QoS classes (rtPS, nrtPS, and BE).

\subsection{Scheduling rtPS, nrtPS and BE Connections}

Each connection i, where $\mathrm{i}$ denotes the connection identification (CID) of rtPS, nrtPS, and BE services, can adopts AMC at the physical layer. Given a prescribed PER $\xi \mathrm{i}$, the SNR thresholds $\{\gamma \mathrm{n}\} \mathrm{N}+1 \mathrm{n}=0$ for connection $\mathrm{i}$ are defined in Section II-C by setting with $\mathrm{P} 0=\xi \mathrm{i}$. Thus, the possible transmission rate (capacity), i.e., number of packets that could be carried by $\mathrm{Nr}$ time slots for connection i at time $\mathrm{t}$ (frame index), can be derived as

$$
C i(t)=N r R i(t)(4)
$$

where $\mathrm{Ri}(\mathrm{t}) \in\{2 \mathrm{Rn}\} \mathrm{Nn}=0$ is the number of packets that can be carried out by one time slot and is determined by channel quality of connection i via AMC as in (1). Notice that either $\mathrm{Ri}(\mathrm{t})$ or $\mathrm{Ci}(\mathrm{t})$ indicates the capacity or channel quality, which will be accounted by the scheduler as we will see next.

At the MAC layer, the scheduler can simply allocates all $\mathrm{Nr}$ time slots per frame to the connection

$$
i^{*}=\arg \max \varphi i(t)(5)
$$

where $\varphi \mathrm{i}(\mathrm{t})$ is the PRF for the connection $\mathrm{i}$ at time $\mathrm{t}$, which will be specified soon. If multiple connections have the same value $\operatorname{maxi}\{\varphi \mathrm{i}(\mathrm{t})\}$, the scheduler can randomly select one of them with even opportunity. For every rtPS connection, the scheduler time-stamps each and every arriving packet according to its arrival time and defines its timeout if the waiting time of such a packet in queue is over on the maximum latency (deadline) Ti. The PRF for an rtPS connection $\mathrm{i}$ at time $\mathrm{t}$ is defined 


\section{International Journal of Science and Research (IJSR) \\ ISSN (Online): 2319-7064}

Index Copernicus Value (2013): 6.14 | Impact Factor (2015): 6.391

$$
\phi_{i}(t)= \begin{cases}\beta_{r t} \frac{R_{1}(t)}{R_{N}} \frac{1}{F_{x}(t)}, & \text { if } F_{i}(t) \geq 1, R_{i}(t) \neq 0 \\ \beta_{r t}, & \text { if } F_{i}(t)<1, R_{i}(t) \neq 0 \\ 0, & \text { if } R_{i}(t)=0\end{cases}
$$

where $\beta r t \in[0,1]$ is the rtPS-class coefficient and $\mathrm{Fi}(\mathrm{t})$ is delay satisfaction indicator, which is defined as

$$
\mathrm{Fi}(\mathrm{t})=\mathrm{Ti}-\Delta \mathrm{T} \mathbf{i}-\mathrm{Wi}(\mathrm{t})+1(7)
$$

with $\Delta \mathrm{Ti} \in[0, \mathrm{Ti}]$ denoting with the guard time region ahead of deadline $\mathrm{Ti}$, and $\mathrm{Wi}(\mathrm{t}) \in[0, \mathrm{Ti}]$ denoting the longest packet waiting time, i.e., the head of line (HOL) delay. If $\mathrm{Fi}(\mathrm{t}) \geq 1$, i.e., $\mathrm{Wi}(\mathrm{t}) \in[0, \mathrm{Ti}-\Delta \mathrm{Ti}]$, the delay requirement was satisfied, and the effect on priority is quantified as $1 / \mathrm{Fi}(\mathrm{i}) \in[0,1]$ : Large values of $\mathrm{Fi}(\mathrm{t})$ indicate high degree of satisfaction, which leads to be low priority. If $\mathrm{Fi}(\mathrm{t})<1$, i.e., $\mathrm{Wi}(\mathrm{t}) \in(\mathrm{Ti}-\Delta \mathrm{Ti}, \mathrm{Ti}]$, the packets of connection $\mathrm{i}$ could be sent immediately to avoid packet drop due to delay outage, so that highest value of PRF $\beta \mathrm{rt}$ is set. Parameter RN := $\operatorname{maxn}\{2 \mathrm{Rn}\} \mathrm{Nn}=0$, and the factor $\mathrm{Ri}(\mathrm{t}) / \mathrm{RN} \in[0,1]$ specifies the normalized channel quality because high received SNR induces high capacity, which results in the high priority. When $\operatorname{Ri}(\mathrm{t})=0$, the channel is in deep fade and capacity is zero, so that connection i should not to be served regardless of delay performance. Notice that the value of $\varphi \mathrm{i}(\mathrm{t})$ for rtPS connection i should lies in $[0, \beta \mathrm{rt}]$.

For every nrtPS connection, guaranteeing the minimum reserved rate $\eta \mathrm{i}$ means, the average transmission rate should be greater than $\eta$ i. In practice to that, if data of connection $i$ are always available in the queue, the average transmission rate at time $t$ is usually estimated over a window size tc based on (4) and (5) as

$$
\hat{\eta}_{i}(t+1)= \begin{cases}\hat{\eta}_{i}(t)\left(1-1 / t_{c}\right), & \text { if } i \neq i^{*} \\ \hat{\eta}_{i}(t)\left(1-1 / t_{c}\right)+C_{i}(t) / t_{c}, & \text { if } i=i^{*} .\end{cases}
$$

We would like to guarantee that ${ }^{\wedge} \eta \mathrm{i}(\mathrm{t}) \geq \eta$ i during the entire service period. The PRF for an nrtPS connection $i$ at time $t$ is defined as

$$
\phi_{i}(t)= \begin{cases}\beta_{\mathrm{nrt}} \frac{R_{i}(t)}{R_{N}} \frac{1}{F_{i}(t)}, & \text { if } F_{i}(t) \geq 1, R_{i}(t) \neq 0 \\ \beta_{\mathrm{nrt}}, & \text { if } F_{i}(t)<1, R_{i}(t) \neq 0 \\ 0, & \text { if } R_{i}(t)=0 .\end{cases}
$$

where $\beta n r t \in[0,1]$ is the nrtPS-class coefficient, and $\operatorname{Fi}(\mathrm{t})$ is the ratio of average transmission rate over the minimum reserved rate

$$
F_{i}(t)=\hat{\eta}(t) / \eta i \cdot(10)
$$

Quantity Fi(t) here is rate satisfaction indicator. If $\operatorname{Fi}(\mathrm{t}) \geq 1$, the rate requirement is satisfied, and its effect on priority is quantified as $1 / \operatorname{Fi}(\mathrm{t}) \in[0,1]$. If $\operatorname{Fi}(\mathrm{t})<1$, the packets of the connection i should be sent as soon as possible to meet the rate requirement; in this case, the upper-bound value of $\beta \mathrm{nrt}$ is set for $\varphi \mathrm{i}(\mathrm{t})$. Once again, the value of $\varphi \mathrm{i}(\mathrm{t})$ lies in $[0, \beta \mathrm{nrt}]$. For BE connections, there are no QoS guarantees. The PRF for a $\mathrm{BE}$ connection $\mathrm{i}$ at time $\mathrm{t}$ is

$$
\phi_{i}(t)=\beta_{\mathrm{BE}} \frac{R_{i}(t)}{R_{N}}
$$

where $\beta B E \in[0,1]$ is the BE-class coefficient. Notice that $\varphi \mathrm{i}(\mathrm{t})$ varies in $[0, \beta \mathrm{BE}]$, which only depends on normalized channel quality regardless of delay or rate performance.

The role of $\beta \mathrm{rtPS}, \beta \mathrm{nrtPS}$, and $\beta \mathrm{BE}$ in (6), (9), and (11), respectively, is to be provide different priorities for different QoS classes. For e.g., if the priority order for different QoS classes is rtPS $>$ nrtPS $>\mathrm{BE}$, the coefficients can be set under the constraint $\beta \mathrm{rtPS}>\beta \mathrm{nrtPS}>\beta \mathrm{BE}$; e.g., $\beta \mathrm{rtPS}=1.0$ $>\beta$ nrtPS $=0.8>\beta \mathrm{BE}=0.6$. Thus, QoS of connections in a high-priority QoS class have be satisfied prior for those of the low-priority QoS class because the value of $\varphi \mathrm{i}(\mathrm{t})$ for QoS unsatisfied connections will equal to the upper-bound $\beta$ rtPS, $\beta$ nrtPS, and $\beta \mathrm{BE}$ for rtPS, nrtPS, and BE connections. The purpose of normalizing $\varphi \mathrm{i}(\mathrm{t})$ in $[0, \beta \mathrm{rtPS}],[0, \beta \mathrm{nrtPS}]$, and $[0, \beta \mathrm{BE}]$, respectively, is to provide comparable priorities among those connections with different kinds of services, which enable exploiting multi user diversity among all those connections with rtPS, nrtPS, and BE services.

In same service class of rtPS or nrtPS, $\varphi$ i(t) for different connections can only depend on the normalized channel quality $\mathrm{Ri}(\mathrm{t}) / \mathrm{RN}$ and QoS satisfaction indicator $\mathrm{Fi}(\mathrm{t})$, where principle is similar to that of the scheme in [6]. However, the major differences with our PRF design is that, the value of ( $\mathrm{i}(\mathrm{t})$ for the QoS unsatisfied connection is set to be its upperbound $\beta$ nrtPS, $\beta r t P S$ and $\beta B E$ for rtPS, nrtPS, and BE connections, respectively. This design can results better QoS guarantees than [6]. For example, when QoS is not satisfied for a connection, bad channel quality may lead to low priority in [6], so that QoS can decrease. However, our design assigns highest priority to such a connection, which increases its QoS as soon as possible, thus providing a better QoS guarantees. A similar observation has been made also in [14] for delay sensitive traffic.

\section{Desirable Features}

In this section, we can summarize the features of our proposed scheduler with reference to the scheduler design criteria suggested in [9]. Here are the attributes of the proposed scheduler are:

1) Efficient bandwidth utilization is achieved through normalized channel quality factor $\mathrm{Ri}(\mathrm{t}) / \mathrm{RN}$ in $\varphi \mathrm{i}(\mathrm{t})$ for each connection, so that scheduler will not assign a time slot to connection with bad channel quality and multiuser diversity can be exploited.

2) Delay bound $\mathrm{Ti}$ is provided for rtPS connections. When HOL delay $\mathrm{Wi}(\mathrm{t})$ approaches $\mathrm{Ti}(\mathrm{Wi}(\mathrm{t}) \in(\mathrm{Ti}-\Delta \mathrm{Ti}, \mathrm{Ti}])$, the highest value $\beta$ rtPS is set for the connection $i$, which will be served as soon as possible. Because wireless channels can experience deep fades, the delay outage event, e.g., $\mathrm{Wi}(\mathrm{t})=\mathrm{Ti}$ and $\mathrm{Ci}(\mathrm{t})=0$, cannot be avoided. Thus, hard delay bound may not be guaranteed.

3) However, controlling of the delay outage probability below the practically acceptable values could be realized by adjusting $\Delta \mathrm{Ti}$.

4) Throughput is guaranteed for nrtPS connections if sufficient bandwidth is provided. When ${ }^{\prime} \eta \mathrm{i}(\mathrm{t})<\eta \mathrm{i}$, is the highest priority $\beta$ nrt will be set for the connection $\mathrm{i}$, which will be served as soon as possible until its throughput requirement is satisfied. 


\section{International Journal of Science and Research (IJSR) \\ ISSN (Online): 2319-7064 \\ Index Copernicus Value (2013): 6.14 | Impact Factor (2015): 6.391}

5) Implementation complexity was very low because our priority-based scheduler simply updates the priority of each connection per frame and allocates $\mathrm{Nr}$ time slots to the connection with the highest priority as in (5).

6) Flexibility is provided because scheduling does not depend on any traffic or channel model.

7) Scalability is achieved. When available bandwidth decreases by adding the new connections to the system for instance, the performance of connections with a lowpriority service classes will be degraded prior to those with a high priority service classes, as we will verify by the simulations in the ensuring section.

\section{Simulations}

Because of the design and performance of fixed TDMA bandwidth allocation for UGS connections are well understood, here, we only focus only on the scheduling for nrtPS, rtPS and BE connections.

We list the assumptions that are employed in simulations.

A1) The wireless channel quality of every connection remains constant per frame, but is allowed to vary from one frame to other frame. This leads to a block-fading channel model, which is suitable for slowly varying wireless channels. Then, AMC is implemented on a frame-by-frame basis [16].

A2) Perfect channel state information (CSI) is available at the receiver side via training-based channel estimation. The corresponding transmission mode selection is fed back to transmitter without error and latency [5]. The assumption is that the feedback channel is error free could be satisfied by using heavily coded feedback streams [3]. On other hand, the feedback latency can be compensated by channel prediction.

A3) Error detection can be based on CRC is perfect, provided that sufficiently reliable error detection CRC codes are used per packet [17].

A4) If a packet was received incorrectly after error detection, we declare it as packet loss.

\subsection{Channel Model}

For fading channels adhering to above A1, the channel quality was captured by single parameter, namely, the instantaneous" SNR $\gamma$, which remains constant during a frame. We support the general Nakagami-m model to describe $\gamma$ statistically [5]. The received SNR $\gamma$ per frame is thus an random variable with a Gamma probability density function, i.e.

$$
p_{\gamma}(\gamma)=\frac{m^{m} \gamma^{m-1}}{\bar{\gamma}^{m} \Gamma(m)} \exp \left(-\frac{m \gamma}{\bar{\gamma}}\right)
$$

where ${ }^{-} \gamma:=\mathrm{E}\{\gamma\}$ is average" received SNR, $\Gamma(\mathrm{m}):=\infty 0$ $\mathrm{tm}-1 \mathrm{e}-\mathrm{tdt}$ is Gamma function, and $\mathrm{m}$ is Nakagami fading parameter $(m \geq 1 / 2)$. This model can includes the Rayleigh channel when $m=1$. A one-to-one mapping between Ricean factor and the Nakagami fading parameter, m allows Ricean channels to be well approximated by the Nakagamim channels [22]. This channel model is more suitable for flatfading channels as well as frequency-selective fading channels come across with orthogonal frequency-division multiplexing (OFDM) systems [12].

With our AMC design in above, the SNR region $[\gamma \mathrm{n}, \gamma \mathrm{n}+1)$ corresponding to transmission mode $n$ establish the channel state indexed by $n$. To describe the transition of those channel states considering mobility-induced Doppler effects, we rely on finite-state Markov chain (FSMC) model. The state transition matrix of such FSMC is

$$
\mathrm{Pc}_{\mathrm{C}}=[\mathrm{Pl}, \mathrm{n}(\mathrm{N}+1) \times(\mathrm{N}+1)(13)
$$

which depends on statistical channel parameters: average received SNR ${ }^{-} \gamma$, Nakagami fading parameter $\mathrm{m}$, and mobility induced Doppler spread fd [15].

Although we adopt channel transition matrix in (13) for the Nakagami fading channel, the ensuing results will apply also to other kinds of channel transition matrices.

\subsection{Parameter Setting}

The wireless channel for connection $\mathrm{i}$ is modeled using an FSMC, as in Section V-A, with parameters: the average received SNR ${ }^{-} \gamma \mathrm{i}$, the Nakagami fading parameter mi, and Doppler frequency fd,i. The frame length for that $\mathrm{Tf}=1 \mathrm{~ms}$. The packet length at the MAC is fixed to be $\mathrm{Nb}=128$ bytes. For every rtPS connection i, we assume that arrival process to the queue is Bernoulli distributed with an given average rate $\eta \mathrm{i}$ and parameter pi $\in(0,1)$. As a result of this, the instantaneous arriving rate at time $\mathrm{t}$ can be derived as

$$
A_{i}(t)= \begin{cases}0, & \text { with probability } p_{i} \\ \eta_{i} /\left(1-p_{i}\right), & \text { with probability } 1-p_{i} .\end{cases}
$$

$\mathrm{T}$

he QoS parameters are PER $\xi \mathrm{i}$ and the maximum delay Ti. We consider that only two rtPS connections are admitted in the system with $\mathrm{i}=1$ and 2 , respectively. Their channel, QoS, and traffic parameters are:

$$
\begin{aligned}
\bar{\gamma}_{1} & =15 \mathrm{~dB}, \quad m_{1}=1.2, \quad f_{d, 1}=10 \mathrm{~Hz}, \quad \xi_{1}=10^{-2} \\
T_{1} & =30 \mathrm{~ms}, \quad \eta_{1}=2 \mathrm{Mbps}, \quad p_{1}=0.4 \\
\bar{\gamma}_{2} & =20 \mathrm{~dB}, \quad m_{2}=1, \quad f_{d, 2}=10 \mathrm{~Hz}, \quad \xi_{2}=10^{-2} \\
T_{2} & =50 \mathrm{~ms}, \quad \eta_{2}=1 \mathrm{Mbps}, \quad p_{2}=0.5 .
\end{aligned}
$$

The guard time is set to be $\Delta \mathrm{Ti}=1 \mathrm{~ms}$. The delay performance of rtPS connections is calculated by the delay outage probability

$$
\delta_{i}(t+1)= \begin{cases}\delta_{i}(t)\left(1-1 / t_{c}\right), & \text { if } W_{i}(t)<T_{i} \\ \delta_{i}(t)\left(1-1 / t_{c}\right)+1 / t_{c}, & \text { if } W_{i}(t)=T_{i} \text { and } i \neq i^{*}\end{cases}
$$

The delay outage event can happens at time $t+1$ when $\mathrm{Wi}(\mathrm{t})$ $=\mathrm{Ti}$ and $\mathrm{i}=\mathrm{i} *$ because $\mathrm{Ci}(\mathrm{t})=0$, or multiple rtPS connections compete with same PRF value $\beta$ rtPS.

For each nrtPS connection i, we assume that data are always available, which is reasonable for FTP applications, for example. The QoS parameters are PER $\xi \mathrm{i}$ and the minimum reserved rate $\eta$ i. Two nrtPS connections are admitted in the 


\section{International Journal of Science and Research (IJSR) \\ ISSN (Online): 2319-7064}

Index Copernicus Value (2013): 6.14 | Impact Factor (2015): 6.391

system with $\mathrm{i}=3$ and 4 . Their channel and QoS parameters are:

$$
\begin{aligned}
& \bar{\gamma}_{3}=15 \mathrm{~dB}, m_{3}=1, f_{d, 3}=10 \mathrm{~Hz}, \xi_{3}=10^{-3}, \eta_{3}=6 \mathrm{Mbps} \\
& \bar{\gamma}_{4}=20 \mathrm{~dB}, m_{4}=1, f_{d, 4}=10 \mathrm{~Hz}, \xi_{4}=10^{-3}, \eta_{4}=3 \mathrm{Mbps} .
\end{aligned}
$$

The rate performance of nrtPS connections is calculated by the average service rate ${ }^{\prime} \mathrm{i}(\mathrm{t})$ over a window size of tc $=$ 1000 ms based on (8).

For each BE connection i, we now assume that data are always available for HTTP or e-mail applications. The pertinent QoS parameter here is just the PER $\xi$ i. We consider two BE connections in system with $\mathrm{i}=5$ and 6 , respectively. Their channel and QoS parameters are as follows:

$$
\begin{array}{llll}
\bar{\gamma}_{5}=16 \mathrm{~dB}, & m_{5}=1, & f_{d, 5}=10 \mathrm{~Hz}, & \xi_{5}=10^{-3} \\
\bar{\gamma}_{6}=18 \mathrm{~dB}, & m_{6}=1, & f_{d, 6}=10 \mathrm{~Hz}, & \xi_{6}=10^{-3} .
\end{array}
$$

The rate performance of $\mathrm{BE}$ connections is also evaluated by average service rate ${ }^{\wedge} \eta \mathrm{i}(\mathrm{t})$ over a window size $\mathrm{tc}=1000 \mathrm{~ms}$ based on (8). The system is simulated over $60000 \mathrm{~ms}$ with bounds $\beta \mathrm{rtPS}=1.0, \beta \mathrm{nrtPS}=0.8$, and $\beta \mathrm{BE}=0.6$.

\section{Conclusion and Future Directions}

At the MAC layer, a cross-layer scheduling algorithm for multiple connections with diverse QoS requirements is developed, which are used in wireless sensor networks, cellular networks and mobile adhoc networks. Each connection admitted in the system is assigned with priority, which is updated dynamically depending upon its channel quality, service priority and QoS satisfaction; thus, the connection with highest priority is scheduled first each time. Our proposed scheduler offers given delay, and rate guarantees for non real-time and real-time traffic; at the same time, it uses wireless bandwidth efficiently by exploiting multi-users diversity among connections with different kinds of services. Further-more, our scheduler enjoys scalability, flexibility and low implementation complexity. Performance of scheduler was evaluated via simulations in the IEEE 802.16 standard setting, where the upper-bound $\beta \mathrm{rtPS}$, $\beta$ nrtPS, $\beta B E$, and the delay guard time $\Delta$ Ti were set to be heuristically.

Their effects on performance are worthy of future research. Furthermore, our scheduler can allocates all $\mathrm{Nr}$ time slots to one connection each time for simplicity; however, scheduling multiple connections every time may lead to better performance, which is under current investigation. The fairness issue for users in the same service class is another topic in our research area. The effects of imperfect channel state information due to estimation error and feedback latency are also more worth further study.

\section{References}

[1] 3GPP TR 25.848 V4.0.0, Physical Layer Aspects of UTRA High Speed Downlink Packet Access (Release 4), 2001.

[2] 3GPP2 C.S0002-0 Version 1.0, Physical Layer Standard for cdma2000 Spread Spectrum Systems, Jul. 1999.
[3] IEEE Standard 802.16 Working Group, IEEE Standard for Local and Metropolitan Area NetworksP art 16: Air Interface for Fixed Broadband Wireless Access Systems (Revision of IEEE Standard 802.16-2001), 2004.

[4] —, FEC Performance With ARQ and Adaptive Burst Profile Selection, 2001.

[5] M. S. Alouini and A. J. Goldsmith, -Alaptive modulation over Nakagami fading channels," Kluwer J. Wireless Commun., vol. 13, no. 1/2, pp. 119-143, May 2000.

[6] M. Andrews, K. Kumaran, K. Ramanan, A. Stolyar, P. Whiting, and R. Vijayakumar, Providing quality of service over a shared wireless link," IEEE Commun. Mag., vol. 39, no. 2, pp. 150-154, Feb. 2001.

[7] A. Doufexi, S. Armour, M. Butler, A. Nix, D. Bull, J. McGeehan, and P. Karlsson, -Acomparison of the HIPERLAN/2 and IEEE 802.11a wireless LAN standards," IEEE Commun. Mag., vol. 40, no. 5, pp. 172-180, May 2002.

[8] S. Falahati, A. Svensson, T. Ekman, and M. Sternad, - Alaptive modulation systems for predicted wireless channels," IEEE Trans. Commun., vol. 52, no. 2, pp. 307-316, Feb. 2004.

[9] H. Fattah and C. Leung, -Anoverview of scheduling algorithms in wireless multimedia networks," IEEE Wireless Commun., vol. 9, no. 5, pp. 76-83, Oct. 2002.

[10] K. J. Hole, H. Holm, and G. E. Oien, - Alaptive multidimensional coded modulation over flat fading channels," IEEE J. Sel. AreasCommun. , vol. 18, no. 7, pp. 1153-1158, Jul. 2000.

[11]E. Hossain and V. K. Bhargava, -Ink-level traffic scheduling for providing predictive QoS in wireless multimedia networks," IEEE Trans. Multimedia, vol. 6, no. 1, pp. 199-217, Feb. 2004.

[12]Z. Kang, K. Yao, and F. Lorenzelli, Nakagami-m fading modeling in the frequency domain for OFDM system analysis,” IEEE Commun. Lett., vol. 7, no. 10, pp. 484-486, Oct. 2003.

[13] J. Karaog uz, High-rate wireless personal area networks," IEEE Commun. Mag., vol. 39, no. 12, pp. 96-102, Dec. 2001.

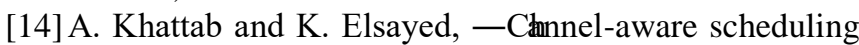
schemes with statistical delay-bound guarantees in wireless multimedia networks," in Proc. ACM/IEEE MSWiM, Venice, Italy, Oct. 4-6, 2004, pp. 31-38.

[15] Q. Liu, S. Zhou, and G. B. Giannakis, -Crss-layer modeling of adaptive wireless links for QoS support in multimedia networks,"

\section{Author Profile}

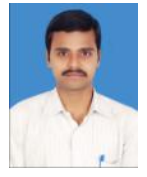

K. Raghavendra Rao, working as assistant professor in Computer Science Engineering from Anurag group of Instituions(Formerly CVSR College of Engineering), Venkatapur(V), Ghatkesar(M), Ranga Reddy District, Hyderabad-500088, Telangana State,

India.

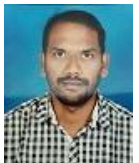

Vadipina Amarnadh, Working as Assistant Professor in computer science and Engineering from Anurag group of Institutions (Formerly CVSR college of Engineering), Venkatapur(V), Ghatkesar(M), Ranga Reddy District, Hyderabad-500088, Telangana State,

India. 